\title{
Avaliação da qualidade da dieta de escolares por seus pais e/ou responsáveis
}

\section{Assessment of the quality of diet of students by their parents and / or caregivers}

Nayara Momm

Doroteia Hofelmann'

' Curso de Nutrição. Universidade do Vale do Itajaí. Itajaí, SC, Brasil.

Correspondência / Correspondence Doroteia Hofelmann

E-mail:doroaph@yahoo.com.br

\section{Resumo}

O objetivo deste trabalho foi caracterizar a avaliação dos pais e responsáveis quanto à qualidade da dieta de crianças matriculadas no $1^{\circ}$ ao $5^{\circ}$ ano, e sua associação com variáveis socioeconômicas, demográficas e comportamentos relacionados à saúde da criança e do responsável. Trata-se de estudo transversal com alunos da rede municipal de Itajaí, Santa Catarina, realizado de junho a agosto de 2011. O perfil socioeconômico, os hábitos relacionados à saúde e o comportamento alimentar foram avaliados por questionário estruturado e de frequência de consumo alimentar (QFCA). Foram avaliadas 523 crianças $(89,9 \%)$, com idade média de 8,5 anos $( \pm 1,52$ anos). Dos responsáveis, $19,3 \%$ (IC 95\% 15,93; $22,8)$ avaliaram como negativa a qualidade da dieta dos escolares. Dentre as crianças que tiveram a dieta percebida pelo responsável como de melhor qualidade, observou-se maior frequência de realização do desjejum $(6,1$ versus 5,2 dias, $\mathrm{p}<0,001)$, e de maior número de refeições ao dia $(5,5$ versus $5,04, \mathrm{p}=0,027$ (IC $95 \%$ : $4,72 ; 5,37)$. Após análise ajustada, a realização de refeições habitualmente em frente à televisão, o consumo de frutas e hortaliças, a presença de constipação, o uso de medicamentos e a avaliação desfavorável da saúde infantil pelo responsável permaneceram associados à percepção mais negativa da dieta pelos pais. Neste estudo, os pais caracterizaram a melhor qualidade alimentar como aquela que contemplou mais alimentos de origem vegetal (alimentos protetores) e foi realizada mais vezes ao dia, associada à melhor avaliação da saúde infantil.

Palavras-chave: Criança. Pais. Saúde Escolar. Hábitos Alimentares. Comportamento Alimentar. 


\section{Abstract}

This study aimed to characterize the evaluation of parents and caregivers of the diet quality of children enrolled in 1st to 5th school year, and it association with socioeconomic and demographic variables, and behaviors related to the child's and parents' health. The cross-sectional study included students from the city of Itajaí, Santa Catarina, between June and August 2011. The socioeconomic profile, health-related habits and eating behavior were assessed by structured questionnaire and food frequency questionnaire (FFQ). We evaluated 523 children (89.9\%) with mean age 8.5 years \pm 1.52 years). Among caregivers, $19.3 \%$ (95\% CI 15.93, 22.8) evaluated the quality of schoolchildren diet as negative. In children who had their diet perceived as better we observed a higher frequency of breakfasts (6.1 versus 5.2 days, $\mathrm{p}<0.001$ ), and greater number of daily meals (5.5 versus 5.04, $\mathrm{p}=0.027$ (95\% CI: 4.72, 5.37). After adjusted analysis, performing usual meals in front of TV, consumption of fruits and vegetables, presence of constipation, use of drugs and unfavorable evaluation of child's health by caregiver, remained associated with a more negative perception of the diet by parents. This study characterized parents' perception about their children's diet as better when it included more vegetable foods (healthy food) and was performed more often in a day, associated to a better evaluation of the child's health.

Key words: Child. Parents. School Health. Food Habits. Feeding Behavior.

\section{Introdução}

A alimentação equilibrada promove a manutenção da saúde. O déficit do consumo alimentar predispõe à desnutrição e à deficiência de micronutrientes; por outro lado, seu excesso associase às doenças crônicas não transmissíveis (DCNT) como hipertensão arterial, diabetes mellitus, dislipidemias e doenças cardiovasculares, ${ }^{1,2}$ principais causas de morte no mundo. ${ }^{3}$

Em 2008, a Pesquisa de Orçamento Familiar identificou que uma em cada três crianças brasileiras de cinco a nove anos apresentava sobrepeso. ${ }^{4}$ Fatores ligados ao ambiente familiar atuam na gênese e na manutenção do excesso de peso. ${ }^{5} \mathrm{O}$ hábito alimentar infantil também sofre influência dos comportamentos e crenças dos adultos em torno delas, das preferências alimentares dos pais, irmãos e colegas e exposição a diversos conteúdos vinculados pela mídia. ${ }^{6}$

O reconhecimento de uma dieta inadequada possibilita o emprego de estratégias que visem corrigir hábitos errôneos e evitar que estes sejam repassados ${ }^{7}$. Para o envolvimento ativo dos 
pais na alteração do comportamento alimentar infantil, eles precisam ser capazes de distinguir a qualidade da dieta e estar conscientes de suas consequências à saúde. Apesar dessa necessidade, dados sobre a avaliação dos pais acerca da qualidade da dieta infantil são praticamente inexistentes, mesmo em publicações internacionais. ${ }^{7}$

Desta forma, esta pesquisa teve por objetivo caracterizar a avaliação dos responsáveis quanto à qualidade da dieta de escolares matriculados na $1^{\mathrm{a}}$ à $5^{\underline{a}}$ série de uma escola de Santa Catarina, e verificar sua associação com variáveis socioeconômicas, demográficas e comportamentos relacionados à saúde, da criança e do responsável.

\section{Metodologia}

Estudo transversal com escolares de uma instituição de ensino municipal de Itajaí, Santa Catarina, realizado nos meses de junho a agosto de 2011. A escola oferecia o ensino do primeiro ao quinto ano, para 627 crianças matriculadas em 2011. O bairro no qual estava inserida era um espaço com nítidas características de segregação social em relação ao centro urbano. O município localiza-se no Vale do Itajaí, a $91 \mathrm{~km}$ da capital do estado, e tem por atividades econômicas de base o porto, o comércio atacadista de combustível e pesca, e o setor de produção industrial. ${ }^{8}$

O cálculo do tamanho amostral foi realizado por meio do aplicativo Epi Info 6.04 (Center of Control of Diseases, EUA). Admitiu-se prevalência de $50 \%$ do desfecho para ampliar o tamanho da amostra, com margem de erro de três pontos percentuais e nível de confiança de $95 \%$, totalizando 395 escolares. Para compensar recusas e permitir maior poder estatístico nas análises entre desfecho e exposições estudadas, acrescentaram-se 30\%, o que totalizou uma amostra de 514 alunos.

Para traçar o perfil socioeconômico e de comportamentos relacionados à saúde e aos hábitos alimentares, utilizou-se um questionário estruturado de frequência de consumo alimentar (QFCA), que foi encaminhado para ser respondido pelo responsável e devolvido em até dez dias.

As variáveis socioeconômicas e demográficas dos responsáveis referiram-se a: escolaridade (anos de estudo concluído $\leq 4$; entre 5 e 8 ou $\geq 9$ ); idade (maior ou menor de 30 anos); condição de posse da moradia (própria, alugada ou cedida) e cor da pele: branca e outras (parda, negra, amarela e indígena) e estado nutricional, por meio do peso e da altura autorreferidos. Da criança foram investigados idade (em anos completos) e o hábito de realizar as refeições com a família e na frente da televisão (não, às vezes ou sempre). Os hábitos relacionados à saúde referiram-se à classificação do responsável quanto à saúde e à alimentação do escolar entre positiva (boa/muito boa/regular) ou negativa (ruim/muito ruim) e atividade física (ocorrência, tipo e frequência) realizada pela criança. Uso de medicamentos e hospitalização da criança no último ano (sim ou não) e presença de constipação (nunca, às vezes ou sempre) também foram questionados aos pais. 
O tempo gasto em atividades de lazer inativo (televisão, computador ou videogame, em número de horas diárias) e as horas semanais de prática esportiva (soma das horas semanais dedicadas a atividades físicas regulares) também foram avaliados. A variável foi categorizada como lazer sedentário quando se assistia à televisão por duas horas ao dia. Foram classificados como fisicamente ativos aqueles que praticavam pelo menos 180 minutos de atividade física na semana, conforme indicação da Academia Americana de Pediatria ${ }^{9}$ e do Colégio Americano de Medicina do Esporte. ${ }^{10}$

Segundo Molina et al., ${ }^{11}$ no Brasil, embora questionários de consumo alimentar tenham sido desenvolvidos e validados, ainda não havia sido proposto nenhum índice que avaliasse a qualidade da alimentação de crianças. Assim, tais autores desenvolveram um questionário com base em estudo semelhante realizado na Espanha com crianças e adolescentes e um índice para avaliação, o Índice de Alimentação do Escolar (ALES). ${ }^{11}$

O QFCA contemplou a frequência de ingestão semanal de feijão, macarrão instantâneo, carne/ frango, peixes e mariscos, batata frita/mandioca ou aipim frito/banana frita, salada crua, batata cozida/mandioca ou aipim cozida, legumes cozidos (menos batata e mandioca/aipim), maionese/ manteiga, hambúrguer/cachorro-quente, leite/iogurte/queijos, frutas, suco de fruta natural, refrigerante, salgados (coxinha, pastel), doces/balas/sobremesa, presunto/salame/mortadela/linguiça e biscoito (chips/recheado), além do ato de realizar o desjejum. ${ }^{11}$

O índice proposto designa uma pontuação segundo frequência da realização do desjejum e consumo de 15 itens alimentares adaptados para as diretrizes da alimentação saudável do Ministério da Saúde. ${ }^{12}$ Assim, optou-se por utilizar o questionário proposto por Molina et al. ${ }^{11}$ Ao considerar o menor tamanho amostral da população investigada no presente estudo, bem como a facilidade de interpretação de variáveis de desfecho binárias, optou-se por empregar a mediana (ao invés do tercil) como ponto de corte para a população investigada.

Para reduzir os itens de consumo alimentar e avaliar sua associação com o desfecho, realizou-se análise fatorial de principal fator, com rotação ortogonal. A adequação da amostra para realização da análise foi avaliada por meio do teste de Kaiser Meyer Olkin, ${ }^{13}$ cujo índice varia de zero a 1,0, tendo borderline de 0,50. O teste de Kaiser Meyer Olkin ${ }^{13}$ atingiu valor global de 0,89 e a análise fatorial de fator principal permitiu o agrupamento dos itens (alimentos) em três dimensões: 1) alimentos ricos em gorduras saturadas, açúcares e sódio: macarrão instantâneo, batata-frita, hambúrguer/cachorro-quente, maionese/manteiga, refrigerante, salgados fritos, doces, embutidos, biscoito recheado e refrigerante; 2) frutas, hortaliças e peixe: peixes e mariscos, salada crua, batata cozida, legumes cozidos, frutas, suco natural de frutas; 3) demais alimentos proteicos: carne/frango, laticínios, feijão. A consistência interna dos itens componentes de cada grupo foi analisada por meio do teste Alpha de Cronbach ${ }^{14}$ e atingiu valores de 0,79 (limite inferior - LI 0,76), 0,74 (LI $0,71)$ e 0,69 (LI 0,65) para os grupos 1,2 e 3, respectivamente. 
As variáveis peso e altura foram obtidas, respectivamente, por meio da balança digital Plenna ${ }^{\circledR}$ e do estadiômetro Seca ${ }^{\circledR}$, seguindo a metodologia de Lohman et al. ${ }^{15}$ Os dados foram tabulados e analisados quanto ao índice de massa corpórea (IMC) de acordo com os percentis para idade, ${ }^{16} \mathrm{com}$ os pontos de corte propostos pelo Ministério da Saúde ${ }^{17}$ tanto para os pais (dados autorreferidos) quanto para as crianças (dados aferidos). Para as análises, foi criada a variável excesso de peso: não (desnutrição e eutrofia), ou sim (sobrepeso e obesidade). A circunferência da cintura foi aferida com uma trena antropométrica inextensível Wiso ${ }^{\circledR}$ no perímetro mais estreito, entre a última costela e a crista ilíaca, sem comprimir os tecidos. ${ }^{18}$

Todos os questionários foram duplamente digitados no aplicativo Microsoft Excel ${ }^{\circledR}$ e conferidos no programa EpiInfo 6.04.

A variável considerada desfecho foi a avaliação da qualidade da dieta dos escolares sob o olhar dos pais: "Como você considera a qualidade da alimentação do seu filho(a)?”. As opções de resposta incluíam as categorias: muito boa, boa, mais ou menos, ruim e muito ruim. Para a análise, as duas primeiras categorias foram agrupadas (positiva), e as três últimas definiram avaliação negativa da percepção dos pais sobre a qualidade da dieta infantil.

A prevalência de qualidade da dieta negativa relatada pelos pais foi comparada entre as categorias das variáveis de exposição por meio das razões de prevalência (RP) e respectivos intervalos de confiança de 95\% (IC 95\%). A análise ajustada foi conduzida por meio da regressão de Poisson, com entrada hierárquica das variáveis no modelo..$^{19}$ Inicialmente, foram incluídas no modelo variáveis do responsável e, posteriormente, variáveis infantis como sexo e faixa etária, seguidas daquelas ligadas ao comportamento alimentar, atividade física e estado nutricional. A presença de constipação e o uso de medicamentos foram em seguida incluídos no modelo; e ao final, a avaliação de saúde pelos pais. Incluíram-se na análise multivariável as variáveis que alcançaram até o nível de confiança de $25 \%$ na análise bivariada, e foram mantidas no modelo final aquelas cujo valor de $\mathrm{p} \leq 0,10 .{ }^{20}$ Adicionalmente, a frequência semanal de consumo alimentar de cada um dos itens componentes do QFCA foi comparada à percepção da qualidade da dieta pelos pais, por meio do teste $t$ de Student. As análises foram realizadas com auxílio do programa Statalo.

A pesquisa foi aprovada pelo Comitê de Ética em Pesquisa da Universidade do Vale do Itajaí, por meio de parecer consubstanciado número 99/1la. A autorização de coleta de dados foi fornecida por meio da assinatura do responsável pela criança no Termo de Consentimento Livre e Esclarecido que detalhava as etapas do estudo.

\section{Resultados}

Foram avaliadas 523 crianças $(89,9 \%)$, sendo $100 \%$ a maior taxa de completude das variáveis para sexo da criança, e $82,2 \%$ a menor para o estado nutricional do responsável. A população 
caracterizou-se homogeneamente quanto ao sexo (50,3\% feminino), com média de idade de 8,5 anos (mínimo 5,8 anos e máximo de 15,1 anos). Dos responsáveis, 83,2\% eram mulheres; a escolaridade mais recorrente para mulheres foi maior ou igual a nove anos, e para os homens foi de até quatro anos completos (tabela 1).

Tabela 1. Descrição das variáveis das crianças e seus respectivos responsáveis, matriculados numa instituição escolar de Itajaí-SC, 2011.

\begin{tabular}{lllll} 
& Variáveis & $\mathrm{n}$ & $\%$ & IC $95 \%$ \\
Categorias & & & & \\
\hline
\end{tabular}

Responsável

Sexo

Masculino

Feminino

Estado nutricional

Baixo-peso

Eutrofia

Sobrepeso

Obesidade

Idade

Até 30 anos

Maior de 30 anos

Cor de pele

Branca

Outras

Escolaridade pai

$$
\begin{aligned}
& \geq 9 \text { anos } \\
& 5-8 \text { anos } \\
& \leq 4 \text { anos }
\end{aligned}
$$

$\begin{array}{lll}83 & 16,8 & 13,7 ; 20,5 \\ 411 & 83,2 & 79,5 ; 86,3\end{array}$

$10 \quad 2,2 \quad 1,1 \quad ; 4,2$

$213 \quad 47,2 \quad 42,6 ; 52,0$

$165 \quad 36,6 \quad 32,2 ; 41,2$

$63 \quad 14,0 \quad 11,0 ; 17,6$

$185 \quad 38,2 \quad 33,9 ; 42,7$

$299 \quad 61,8 \quad 57,3 ; 66,1$

$336 \quad 67,1 \quad 62,7 ; 71,1$

$165 \quad 32,9 \quad 28,9 ; 37,3$

$\begin{array}{lll}141 & 29,1 & 25,1 ; 33,4 \\ 203 & 41,9 & 37,4 ; 46,4 \\ 141 & 29,1 & 25,1 ; 33,4\end{array}$




\begin{tabular}{|c|c|c|c|}
\hline Categorias & $\mathrm{n}$ & $\%$ & IC $95 \%$ \\
\hline \multicolumn{4}{|l|}{ Escolaridade mãe } \\
\hline$\geq 9$ anos & 185 & 37,0 & 32,$8 ; 41,4$ \\
\hline $5-8$ anos & 224 & 44,8 & 40,$4 ; 49,3$ \\
\hline$\leq 4$ anos & 91 & 18,2 & 15,$0 ; 21,9$ \\
\hline \multicolumn{4}{|l|}{ Criança } \\
\hline \multicolumn{4}{|l|}{ Sexo } \\
\hline Masculino & 260 & 49,7 & 45,$4 ; 54,1$ \\
\hline Feminino & 263 & 50,3 & 45,$9 ; 54,6$ \\
\hline \multicolumn{4}{|l|}{ Idade } \\
\hline 6 a 9 anos & 419 & 80,1 & 76,$7 ; 83,5$ \\
\hline 10 anos ou mais & 104 & 19,9 & 16,$5 ; 23,3$ \\
\hline \multicolumn{4}{|l|}{ Estado nutricional } \\
\hline Baixo-peso & 12 & 2,4 & 1,$3 ; 4,2$ \\
\hline Eutrofia & 341 & 67,7 & 63,$4 ; 71,7$ \\
\hline Sobrepeso & 93 & 18,5 & 15,$2 ; 22,2$ \\
\hline Obesidade & 58 & 11,5 & 8,$9 ; 14,7$ \\
\hline \multicolumn{4}{|l|}{ Lazer sedentário } \\
\hline Até 2 horas/dia & 226 & 72,2 & 66,$8 ; 77,0$ \\
\hline Mais de 2 horas/dia & 87 & 27,8 & 23,$0 ; 33,2$ \\
\hline \multicolumn{4}{|c|}{ Avaliação de saúde pelo responsável } \\
\hline Positiva & 465 & 89,4 & 86,$4 ; 91,9$ \\
\hline Negativa & 55 & 10,6 & 8,$1 ; 13,6$ \\
\hline \multicolumn{4}{|c|}{ Avaliação da alimentação pelo responsável } \\
\hline Positiva & 417 & 80,7 & 76,$9 ; 83,9$ \\
\hline Negativa & 100 & 19,3 & 16,$1 ; 23,1$ \\
\hline
\end{tabular}

IC 95\%= intervalo de confiança de 95\% 
A maior parte dos pais avaliou positivamente a qualidade da dieta $(80,7 \%$ IC 95\% 76,9; 83,9), enquanto 19,3\% (IC 95\% 16,1; 23,1) a classificaram de forma mais negativa (tabela 1). As crianças que tiveram sua dieta percebida pelo responsável como de melhor qualidade também tiveram maior frequência de realização do desjejum $(6,1$ versus 5,2 dias, $\mathrm{p}<0,001)$ e maior número de refeições ao dia $(5,5$ versus $5,04, \mathrm{p}=0,027)$.

O hábito de realizar mais refeições com a família também se mostrou associado ao desfecho (RP 1,56 IC 95\% 0,98; 2,47). Já as crianças que realizavam as refeições sempre em frente à televisão apresentaram prevalência 79\% (RP 1,79 IC 95\% 1,21; 2,64) superior às que tiveram sua alimentação considerada como de pior qualidade pelos pais. Os escolares que tiveram sua dieta considerada de pior qualidade pelos pais apresentaram maior prevalência de uso de medicamentos no último ano, de serem constipados, bem como menor prática de atividade física no lazer, e tiveram sua saúde avaliada pelos pais mais negativamente (tabela 2).

Tabela 2. Associação de variáveis da criança e avaliação negativa da qualidade da dieta pelos responsáveis. Estudo com alunos matriculados em instituição escolar de Itajaí-SC, 2011.

\begin{tabular}{|c|c|c|c|c|c|c|}
\hline $\begin{array}{l}\text { Variáveis } \\
\text { Categorias }\end{array}$ & $\mathrm{n}$ & $\%$ & RP bruta (IC 95\%) & $\mathrm{p}^{*}$ & $\begin{array}{l}\text { RP ajustada } \\
\text { (IC 95\%) }\end{array}$ & $\mathrm{p}^{* *}$ \\
\hline \multicolumn{7}{|l|}{ Responsável } \\
\hline Sexo do avaliador & & & & 0,588 & & \\
\hline Masculino & 14 & 16,9 & 1,00 & & & \\
\hline Feminino & 79 & 19,5 & $1,15(0,69 ; 1,94)$ & & & \\
\hline Faixa etária & & & & 0,515 & & \\
\hline Até 30 anos & 33 & 18,0 & 1,00 & & & \\
\hline Maior de 30 anos & 61 & 20,5 & $1,13(0,77 ; 1,66)$ & & & \\
\hline \multicolumn{7}{|l|}{ Escolaridade do pai } \\
\hline$\geq 9$ anos & 25 & 17,9 & 1,00 & 0,648 & & \\
\hline $5-8$ anos & 39 & 19,4 & $0,97(0,63 ; 1,50)$ & & & \\
\hline$\leq 4$ anos & 28 & 20,0 & $0,89(0,55 ; 1,45)$ & & & \\
\hline Escolaridade da mãe & & & & 0,286 & & \\
\hline$\geq 9$ anos & 40 & 21,9 & 1,00 & & & \\
\hline $5-8$ anos & 43 & 19,4 & $1,18(0,69 ; 2,01)$ & & & \\
\hline$\leq 4$ anos & 15 & 16,5 & $1,33(0,77 ; 2,27)$ & & & \\
\hline
\end{tabular}




\begin{tabular}{|c|c|c|c|c|c|c|}
\hline $\begin{array}{l}\text { Variáveis } \\
\text { Categorias }\end{array}$ & $\mathrm{n}$ & $\%$ & RP bruta (IC 95\%) & $p^{*}$ & $\begin{array}{l}\text { RP ajustada } \\
\text { (IC 95\%) }\end{array}$ & $\mathrm{p}^{* *}$ \\
\hline Estado nutricional & & & & 0,690 & & \\
\hline Eutrofia & 40 & 18,1 & 1,00 & & & \\
\hline Sobrepeso & 34 & 20,7 & $1,15(0,76 ; 1,73)$ & & & \\
\hline Obesidade & 12 & 19,1 & $1,05(0,59 ; 1,88)$ & & & \\
\hline Criança & & & & & & \\
\hline Sexo & & & & 0,258 & & \\
\hline Masculino & 55 & 21,3 & 1,00 & & & \\
\hline Feminino & 45 & 17,4 & $0,82(0,57 ; 1,16)$ & & & \\
\hline Faixa etária & & & & 0,632 & & \\
\hline Até 9 anos & 82 & 19,8 & 1,00 & & & \\
\hline 10 ou mais & 18 & 17,7 & $0,89(0,56 ; 1,42)$ & & & \\
\hline Refeições com a família & & & & 0,061 & & \\
\hline $1-3 \operatorname{dias} / \mathrm{sem}$ & 16 & 27,6 & $1,56(0,98 ; 2,47)$ & & & \\
\hline $4-7$ dias/sem & 80 & 17,7 & 1,00 & & & \\
\hline $\begin{array}{l}\text { Refeições em frente à } \\
\text { televisão }\end{array}$ & & & & 0,003 & & 0,040 \\
\hline Nunca ou às vezes & 73 & 17,1 & 1,00 & & 1,00 & \\
\hline Sempre & 25 & 30,5 & $1,79(1,21 ; 2,64)$ & & $1,43(1,00 ; 2,05)$ & \\
\hline $\begin{array}{l}\text { Consumo de alimentos } \\
\text { ricos em gordura } \\
\text { saturada, açúcares e } \\
\text { sódio }\end{array}$ & & & & 0,374 & & \\
\hline $0,0-1,4 \mathrm{x} / \mathrm{sem}$ & 38 & 20,3 & 1,00 & & & \\
\hline $1,5-2,6 \mathrm{x} / \mathrm{sem}$ & 34 & 21,1 & $1,04(0,69 ; 1,57)$ & & & \\
\hline $2,7-6,7 \mathrm{x} / \mathrm{sem}$ & 28 & 16,6 & $0,82(0,52 ; 1,27)$ & & & \\
\hline $\begin{array}{l}\text { Consumo de frutas, } \\
\text { hortaliças e peixes }\end{array}$ & & & & $<0,001$ & & $<0,001$ \\
\hline $0,0-4,3 \times / \mathrm{sem}$ & 66 & 35,5 & 1,00 & & 1,00 & \\
\hline $4,4-6,0 \mathrm{x} / \mathrm{sem}$ & 21 & 12,4 & $0,35(0,22 ; 0,55)$ & & $0,38(0,24 ; 0,59)$ & \\
\hline $6,1-7,0$ x/sem & 13 & 8,0 & $0,23(0,13 ; 0,39)$ & & $0,24(0,14 ; 0,42)$ & \\
\hline
\end{tabular}




\begin{tabular}{|c|c|c|c|c|c|c|}
\hline $\begin{array}{l}\text { Variáveis } \\
\text { Categorias }\end{array}$ & $\mathrm{n}$ & $\%$ & RP bruta (IC 95\%) & $\mathrm{p}^{*}$ & $\begin{array}{l}\text { RP ajustada } \\
\text { (IC 95\%) }\end{array}$ & $\mathrm{p}^{* *}$ \\
\hline $\begin{array}{l}\text { Consumo dos demais } \\
\text { alimentos proteicos }\end{array}$ & & & & $<0,001$ & & \\
\hline $0,0-1,5 \mathrm{x} / \mathrm{sem}$ & 54 & 28,1 & 1,00 & & & \\
\hline $1,6-2,8 \mathrm{x} / \mathrm{sem}$ & 29 & 15,6 & $0,55(0,37 ; 0,83)$ & & & \\
\hline $2,9-6,3 \mathrm{x} / \mathrm{sem}$ & 17 & 12,2 & $0,43(0,26 ; 0,72)$ & & & \\
\hline Fisicamente sedentário & & & & 0,038 & & \\
\hline Sim & 33 & 15,1 & 1,00 & & & \\
\hline Não & 67 & 22,5 & $1,49(1,02 ; 2,18)$ & & & \\
\hline Obesidade & & & & 0,140 & & \\
\hline Não & 81 & 18,4 & 1,00 & & & \\
\hline Sim & 15 & 26,3 & $1,43(0,89 ; 2,30)$ & & & \\
\hline Constipação & & & & $<0,001$ & & $<0,001$ \\
\hline Não & 58 & 14,7 & 1,00 & & 1,00 & \\
\hline Às vezes/sempre & 40 & 33,6 & $2,28(1,61 ; 3,23)$ & & $1,83(1,30 ; 2,57)$ & \\
\hline Uso medicamento & & & & 0,001 & & 0,008 \\
\hline Não & 25 & 11,7 & 1,00 & & 1,00 & \\
\hline Sim & 71 & 24,3 & $2,07(1,36 ; 3,15)$ & & $1,73(1,16 ; 2,58)$ & \\
\hline Hospitalização & & & & 0,761 & & \\
\hline Não & 93 & 19,6 & 1,00 & & & \\
\hline Sim & 5 & 17,2 & $0,88(0,39 ; 2,00)$ & & & \\
\hline $\begin{array}{l}\text { Avaliação da saúde pelo } \\
\text { responsável }\end{array}$ & & & & $<0,001$ & & $<0,001$ \\
\hline Positiva & 71 & 15,4 & 1,00 & & 1,00 & \\
\hline Negativa & 28 & 50,9 & $3,30(2,36 ; 4,63)$ & & $1,99(1,38 ; 2,87)$ & \\
\hline
\end{tabular}

$\mathrm{RP}=$ razão de prevalência; $\mathrm{x} / \mathrm{sem}=$ vezes na semana; IC 95\%= intervalo de confiança de 95\%; *teste de Wald; ** ajustada para variáveis com valor de $\mathrm{p}<0,10$. 
Nenhuma das variáveis do responsável associou-se estatisticamente com a prevalência de classificar mais negativamente a dieta infantil. Entre os filhos de mães com menor escolaridade, observou-se uma propensão à dieta classificada como de pior qualidade (tabela 2). Dentre as variáveis ligadas ao hábito alimentar, o maior consumo de frutas e hortaliças, bem como de alimentos proteicos, esteve positivamente associados à classificação da dieta como de melhor qualidade; contudo, o consumo de alimentos ricos em gordura saturada, açúcar e sódio não esteve associado à avaliação negativa da qualidade da dieta sob o olhar dos responsáveis (tabela 2).

Após análise ajustada, a realização de refeições habitualmente em frente à televisão, o consumo de frutas e hortaliças, a presença de constipação, o uso de medicamentos e a avaliação desfavorável da saúde infantil permaneceram associados à avaliação mais negativa da dieta pelos pais. Crianças que sempre realizavam as refeições em frente à televisão apresentaram prevalência $43 \%$ maior de terem sua dieta classificada como de pior qualidade (tabela 2).

A análise individual da média de consumo de cada alimento, de acordo com o questionário, foi significativa para: maior consumo de carnes, batata-frita, salada crua, tubérculos cozidos, legumes, frutas, suco de frutas natural e feijão, entre os escolares que tiveram sua dieta classificada como de melhor qualidade pelos pais (tabela 3). 
Tabela 3. Médias e desvios-padrão de consumo por alimento segundo a avaliação da qualidade da dieta dos responsáveis pelas crianças matriculadas em instituição escolar de Itajaí-SC, 2011.

\begin{tabular}{|c|c|c|c|}
\hline \multirow{3}{*}{ Alimentos } & \multicolumn{3}{|c|}{ Avaliação da qualidade da dieta } \\
\hline & $\begin{array}{l}\text { Positiva } \\
(\mathrm{n}=417)\end{array}$ & $\begin{array}{c}\text { Negativa } \\
(\mathrm{n}=100)\end{array}$ & $\mathrm{p}^{*}$ \\
\hline & Média $(\underline{\mathrm{DP}})$ & Média $(\underline{\mathrm{DP}})$ & \\
\hline Macarrão instantâneo & $1,26(1,66)$ & $1,20(1,52)$ & 0,726 \\
\hline Carne/frango & $4,56(2,32)$ & $3,71(2,65)$ & $<0,001$ \\
\hline Peixes e mariscos & $0,79(1,26)$ & $0,71(1,47)$ & 0,542 \\
\hline $\begin{array}{l}\text { Batata frita/mandioca ou aipim frito/ } \\
\text { banana frita }\end{array}$ & $1,66(1,94)$ & $1,22(1,51)$ & 0,034 \\
\hline Vegetais crus & $3,11(2,61)$ & $1,73(2,06)$ & $<0,001$ \\
\hline $\begin{array}{l}\text { Batata cozida/mandioca ou aipim } \\
\text { cozido }\end{array}$ & $2,24(2,01)$ & $1,26(1,76)$ & $<0,001$ \\
\hline Legumes cozidos & $2,18(2,36)$ & $1,03(1,68)$ & $<0,001$ \\
\hline Maionese/manteiga & $2,48(2,53)$ & $2,35(2,51)$ & 0,633 \\
\hline Hambúrguer/cachorro quente & $1,29(1,61)$ & $1,32(1,64)$ & 0,869 \\
\hline Leite/Iogurte/queijos & $4,85(2,57)$ & $4,42(2,70)$ & 0,136 \\
\hline Frutas & $4,14(2,59)$ & $2,69(2,53)$ & $<0,001$ \\
\hline Suco de fruta natural & $2,19(2,49)$ & $1,15(1,90)$ & $<0,001$ \\
\hline Refrigerante & $2,04(1,84)$ & $1,93(1,53)$ & 0,626 \\
\hline Salgados (coxinha, pastel) & $1,25(1,72)$ & $1,36(1,97)$ & 0,566 \\
\hline Doces/balas/sobremesa & $3,17(2,46)$ & $2,82(2,42)$ & 0,200 \\
\hline Presunto/salame/mortadela/linguiça & $2,58(2,36)$ & $2,21(1,74)$ & 0,152 \\
\hline Biscoito (chips/recheado) & $3,43(3,18)$ & $3,45(2,94)$ & 0,596 \\
\hline Feijão & $5,02(2,45)$ & $4,15(2,70)$ & 0,002 \\
\hline
\end{tabular}

*Test $\mathrm{t}$ de Student 


\section{Discussão}

A maioria dos responsáveis pelas crianças avaliadas classificou a qualidade da dieta de seus filhos como positiva (80,7\%). Kourlaba et al., ${ }^{7}$ ao estudarem a percepção de mães sobre a alimentação de seus filhos entre dois e cinco anos, verificaram que $82,5 \%$ delas superestimavam a qualidade da dieta infantil e seus filhos tiveram maior ingestão de legumes, carne e leite, comparados àqueles cujas mães avaliaram corretamente a qualidade da dieta.

Entre as crianças investigadas, observou-se associação significativa entre a avaliação da qualidade da dieta indicada pelos pais e aquela classificada por meio do índice de qualidade da dieta. Dentre os itens que compõem o índice avaliado, o consumo de frutas e hortaliças, feijão e alimentos proteicos, bem como a realização frequente do desjejum, estiveram associados à avaliação mais positiva da dieta infantil pelos pais.

Outros autores também observaram que frutas e hortaliças são alimentos reconhecidos e enfatizados por jovens e adultos ao construírem o conceito de alimentação saudável. ${ }^{21,22}$ Os pais têm importante papel na promoção do consumo de frutas e legumes, e a ingestão desses alimentos por crianças pode estar vinculada ao conhecimento da mãe e a sua conduta em relação à alimentação saudável. ${ }^{23}$ A apresentação de conceitos adequados referentes a uma dieta saudável pelos genitores pode estar ligada à divulgação das informações de alimentação e nutrição nos meios de comunicação. ${ }^{22}$ Muitos pais têm dificuldades para avaliar os itens alimentares e as proporções indicadas para uma alimentação saudável. ${ }^{24}$ No decorrer da educação, alguns pais acreditam que ensinar os filhos a não jogar comida fora pode transmitir o princípio do autocontrole e autorregulação em relação à comida. No entanto, há pais que não permitem que as crianças escolham as quantidades que queiram comer; assim, as crianças são induzidas a se adaptar às quantidades desejadas pelos pais, obedecendo e respeitando suas regras. ${ }^{25}$ Além disso, a imagem ainda bastante disseminada, que associa excesso de peso infantil e saúde, ${ }^{26}$ pode ter influenciado esse resultado. Observa-se que o conceito de "comer bem" para pais e/ou responsáveis pode privilegiar aspectos quantitativos em detrimento da qualidade daquilo que é ingerido.

O hábito de realizar as refeições com a família está positivamente associado com a ingestão de alimentos saudáveis e inversamente com a prevalência de excesso de peso. ${ }^{27}$ Já o hábito de comer enquanto se assiste à televisão está associado positivamente às dietas menos saudáveis e ao excesso de peso. ${ }^{6}$ No presente estudo, o consumo alimentar habitual em frente à televisão mantevese associado à pior percepção dos pais sobre a qualidade da dieta. A publicidade de alimentos veiculada na televisão pode ser interpretada por algumas mães como fator de impacto negativo na alimentação de seus filhos. ${ }^{6}$ 
Na presente pesquisa, desfechos negativos de saúde, como sedentarismo pela inatividade física, maior propensão a episódios de constipação, uso de remédios no ano anterior e saúde percebida pelos pais como mais negativa também foram mais frequentes entre os escolares com dieta considerada ruim pelos pais.

Estudos apontam para a existência de um agrupamento de fatores de risco desde a infância e seu compartilhamento no ambiente familiar. ${ }^{28,29} \mathrm{O}$ padrão de inatividade física é frequentemente encontrado em crianças com hábitos alimentares inadequados. ${ }^{28} \mathrm{O}$ hábito alimentar tem impacto na saúde e nutrição; a baixa ingestão de frutas, verduras de folhas, legumes e carnes repercute na baixa ingestão de micronutrientes e, por consequência, em deficiências nutricionais ${ }^{30}$ que favorecem o surgimento de algumas doenças. No presente estudo, contudo, não é possível afirmar se a avaliação negativa da saúde pelos pais foi decorrente da má alimentação dos filhos, tenha implicado na piora da saúde destes, ou se crianças com outros comportamentos ruins para a saúde também possuem pior alimentação.

Os produtos mais frequentemente associados pelos pais a uma dieta saudável, no estudo de Lopez-Dicastillo et al. ${ }^{25}$ foram: frutas, verduras, legumes, peixes, produtos lácteos e azeite de oliva. Os genitores ainda definiram a dieta saudável como aquela variada, com equilíbrio entre os grupos alimentares e limitado consumo de sal, açúcar e gordura, assim como restrita em alguns produtos, como doces, balas, produtos industriais de panificação e fast food. ${ }^{22}$

A frequência do consumo de alimentos ricos em gorduras saturadas, açúcares e sódio não esteve associada à avaliação da dieta infantil pelos pais neste estudo. Alimentos densamente energéticos são muitas vezes utilizados pelos pais como sistema de recompensa para o bom comportamento infantil. ${ }^{31}$ Em estudos com mães mexicanas de baixa renda, Jimenez-Cruz et al. ${ }^{32}$ observaram que elas não consideraram que refrigerantes e bebidas açucaradas ou lanches gordurosos poderiam ser prejudiciais à saúde de seus filhos.

O delineamento transversal do estudo limita conclusões relacionadas à direção causal das associações observadas, mas a relação entre desfecho e muitas das variáveis analisadas é bidirecional. A escassez de estudos sobre a percepção dos pais quanto à alimentação de seus filhos dificultou a comparação dos resultados. Além disso, a dependência da memória e interpretação dos mesmos para preenchimento do questionário pode ter influenciado os resultados.

Para minimizar equívocos e perdas, a pesquisadora manteve-se disponível na escola para responder às dúvidas; foram realizadas, ainda, orientações verbais e escritas quanto ao preenchimento do material repassado às professoras e aos escolares. 


\section{Conclusões}

A avaliação prevalente dos responsáveis foi de uma dieta de boa qualidade. O desfecho esteve associado ao maior consumo de frutas, verduras e alimentos proteicos, número de refeições diárias e realização do desjejum. As dietas classificadas como de pior qualidade estiveram associadas aos hábitos de comer em frente à televisão, consumir poucas frutas e hortaliças, possuir constipação, ter feito uso de medicamentos no ano anterior e a avaliação desfavorável da saúde. Das variáveis dos responsáveis, nenhuma se manteve associada.

A conduta alimentar infantil pode ser influenciada por seus pais; assim, estes deveriam fazer parte do público-alvo nas educações nutricionais. Salienta-se que a realização de futuros trabalhos avaliando o conhecimento dos pais acerca das recomendações nutricionais, bem como seus comportamentos como formadores do hábito alimentar infantil, proporcionaria subsídios a estratégias nutricionais em escala nacional.

\section{Referências}

1. Mondini L, Monteiro CA. Mudanças no padrão de alimentação da população urbana brasileira (19621988). Rev Saúde Públ. 1994;28(6):433-39.

2. Conceição SIO, Santos CJN, Silva AAM, Silva JS, Oliveira TC. Consumo alimentar de escolares das redes pública e privada de ensino em São Luís, Maranhão. Rev Nutr. 2010;23(6):993-04.

3. Malta DC, Morais Neto OL, Silva Junior, JB. Apresentação do plano de ações estratégicas para o enfrentamento das doenças crônicas não transmissíveis no Brasil, 2011 a 2022. Epidemiol Serv Saúde. 2011;20(4):425-38.

4. IBGE. POF 2008-2009: desnutrição cai e peso das crianças brasileiras ultrapassa padrão internacional [Internet]. 2010. [citado em 2012 ago 03]. Disponível em: <http://www.ibge.gov.br/home/presidencia/ noticias/noticia_visualiza.php?id_noticia=1699\&id_pagina=1/>.

5. Ramos AMPP, Barros Filho AA. Prevalência da obesidade em adolescentes de Bragança Paulista e sua relação com a obesidade dos pais. Arq Bras Endocrinol Metab. 2003;47(6):663-68.

6. Yu JH. Mothers' perceptions of the negative impact on TV food ads on children's food choices. Appetite. 2012;59(2):372-6.

7. Kourlaba G, Kondaki K, Grammatikaki E, Roma-Giannikou E, Manios Y. Diet quality of preschool children and maternal perceptions/misperceptions: The GENESIS study. BMC Public Health. 2009;123(11):738-42.

8. Secretaria de Estado de Desenvolvimento Regional. Itajaí [Internet]. 2010 [citado em 2012 out 05]. Disponível em: $<$ http://www.iai.sdr.sc.gov.br/index2.php?option=com_content\&do_pdf=1\&id=70>. 
9. Flores PT, Lima K, Pucci V, Souza JG, Nornberg ML, Benedetti FJ. Influência do tempo em frente à televisão na obesidade e no consume alimentar em crianças e adolescentes. 2012. Disponível em: $<$ http://www.unifra.br/eventos/sepe2012/Trabalhos/6244.pdf>

10. American College of Sports Medicine. Guidelines for exercise testing and prescription. Philadelphia: Lea \& Febiger; 1991.

11. Molina MCB, Lopéz PM, Faria CP, Cade NV, Zandonade E. Preditores socioeconômicos da qualidade da alimentação de crianças. Rev Saúde Públ. 2010; 44:785-92.

12. Brasil. Ministério da Saúde. Guia alimentar para a população brasileira: promovendo a alimentação saudável. Brasília: Ministério da Saúde; 2008.

13. Kaiser HF. An index of factorial simplicity. Psychometrika. 1974;39:31-6.

14. Cronbach LJ. Coefficient alpha and the internal structure of the tests. Psychometrika. 1951;16(3):297-34.

15. Lohman T, Roche A, Martorell R. Anthropometric Standardization Reference Manual. Champaign: Human Kinetics Books; 1988.

16. World Health Organization. Growth reference data for 5-19 years [Internet]. 2007 [citado em 2012 jul 30]. Disponível em: <http://www.who.int/growthref/en/>.

17. Brasil. Ministério da Saúde Brasil. Vigilância Alimentar e Nutricional. SISVAN: Orientações para a coleta e análise de dados antropométricos em serviços de saúde - Norma Técnica - SISVAN. Brasília: Ministério da Saúde; 2008.

18. Taylor RW, Jones IE, Williams SM, Goulding A. Evaluation of waist circumference, waist-to-hip ratio, and the conicity index as screening tools for high trunk fat mass, as measured by dual-energy X-ray absorptiometry, in children aged 3-19 y. Am J Clin Nutr. 2000;72(2):490-95.

19. Victora CG, Huttly SR, Fuchs SC, Olinto MT. The role of conceptual frameworks in epidemiological analysis: a hierarchical approach. Int J Epidemiol. 1997;26(1):224-7.

20. Kirkwood B. Essentials of medical statistics. Oxford: Blackwell; 1988.

21. Paquette MC. Perceptions of healthy eating: state of knowledge and research gaps. Can J Public Health. 2005;96(Suppl 3):S15-9.

22. Toral N, Conti MA, Slater B. A alimentação saudável na ótica dos adolescentes: percepções e barreiras à sua implementação e características esperadas em materiais educativos. Cad Saúde Pública 2009;25(11):2386-94.

23. Yung TK, Lee A, Ho MM, Keung VM, Lee JC. Maternal influences on fruit and vegetable consumption of schoolchildren: case study in Hong Kong. Matern Child Nutr. 2010;6(2):190-8.

24. Barbosa, RMS, Colares LGT, Soares EA. Percepção de responsáveis e recreadores sobre diferentes representações gráficas de guia alimentar para crianças de dois a três anos. Rev Paul Pediatr. 2008;26(4):350-6.

25. Lopez-Dicastillo O, Grande G, Callery P. Parents' contrasting views on diet versus activity of children: Implications for health promotion and obesity prevention. Patient Educ Couns. 2010;78(1):117-23. 
26. Campbell MW, Williams J, Hampton A, Wake M. Maternal concern and perceptions of overweight in Australian preschool- aged children. Med J Aust. 2006; 184(6):274-7.

27. Levy RB, Castro IRR, Cardoso LO, Tavares LF, Sardinha LMV, Gomes FS et al. Consumo e comportamento alimentar entre adolescentes brasileiros: Pesquisa Nacional de Saúde do Escolar (PeNSE), 2009. Ciênc Saúde Colet. 2010;15(Suppl.2):S3085-97.

28. Pearson N., Biddle SJ. Sedentary behavior and dietary intake in children, adolescents, and adults. A systematic review. Am J Prev Med. 2011:41(2):178-88.

29. Gubbels JS, Kremers SPJ, Stafleu A, Dagnelie PC, Vries SI, Vries NK et al. Clustering of Dietary Intake and Sedentary Behavior in 2-Year-Old Children. J Pediatr. 2009;155(2):194-8.

30. Bortolini GA, Gubert MB, Santos LMP. Consumo alimentar entre crianças brasileiras com idade de 6 a 59 meses. Cad Saúde Pública. 2012;28(9):1759-71.

31. Crawford DA, Balla K, Clelanda VJ, Campbella KJ, Timperioa AF, Abbotta G. et al. Home and neighbourhood correlates of BMI among children living in socioeconomically disadvantaged neighbourhoods. BJN. 2012;107(7):1028-36.

32. Jimenez-Cruz A, Bacardi-Gascon M, Castillo-Ruiz O, Mandujano-Trujillo Z, Pichardo-Osuna A. Low income, Mexican mothers' perception of their infants' weight status and beliefs about their foods and physical activity. Child Psychiatry Hum Dev. 2010;41(5):490-00. 
